\title{
Labourer la terre, tisser la vie. Éclats d'analogies dans les Andes sud-péruviennes
}

\section{Ingrid Hall}

\section{Q OpenEdition}

\section{Journals}

Édition électronique

URL : https://journals.openedition.org/jsa/12162

DOI : 10.4000/jsa.12162

ISSN : 1957-7842

Éditeur

Société des américanistes

\section{Édition imprimée}

Date de publication : 31 juillet 2012

Pagination : 101-131

ISSN : 0037-9174

\section{Référence électronique}

Ingrid Hall, «Labourer la terre, tisser la vie. Éclats d'analogies dans les Andes sud-péruviennes », Journal de la Société des américanistes [En ligne], 98-1 | 2012, mis en ligne le 10 juin 2015, consulté le 03 septembre 2022. URL : http://journals.openedition.org/jsa/12162 ; DOI : https://doi.org/10.4000/ jsa. 12162 


\title{
LABOURER LA TERRE, TISSER LA VIE. ÉCLATS D'ANALOGIES DANS LES ANDES SUD-PÉRUVIENNES
}

\author{
Ingrid HALL *
}

\begin{abstract}
À partir d'une étude ethnographique de la mise en culture d'une parcelle irriguée réalisée dans une communauté paysanne des Andes sud-péruviennes (Calca, Cusco), différentes analogies établies par les paysans ont pu être mises en évidence, tout au long du cycle agricole, entre cette culture irriguée et des domaines relevant notamment de la physiologie humaine et du tissage. Dans cet article, nous présenterons ces analogies en suivant le déroulement des actions agricoles. Notre propos n'est pas tant de livrer une interprétation que de réfléchir à la structuration des analogies entre elles. Pour cela, nous utiliserons le concept de « schème générateur de la pratique » de Bourdieu (1980) et celui de "schème intégrateur » de Descola (2005). [Mots-clés : techniques agricoles, tissage, physiologie humaine, concept de schème, Andes, Pérou.]
\end{abstract}

Plough the earth, weave the life. Sparkles of analogies in the South Peruvian Andes. The ethnographic datum analyzed in this paper concerns the cultivation of an irrigated plot in a peasant community of the South Peruvian Andes (Calca, Cusco). We will focus on the analogies made by peasants, which relate to two specific semantic domains : human physiology and weaving techniques. We propose a presentation of each punctual analogy according to the agricultural cycle. Interpretation is not our main proposal as we are more interested in the way analogies are structured between them. We will use the concept of schemata defined by Bourdieu (1980) as " generator of practice» and Descola (2005) as «integrator». [Key words : analogies, agricultural techniques, weaving, human physiology, concept of schemata, Andes, Peru.]

Labrar la tierra, tejer la vida. Fragmentos de analogías en los Andes sur peruanos. A partir de la etnografía del cultivo de una parcela irrigada en una comunidad del sur del Perú (Calca, Cusco) expondremos las diferentes analogías que sugieren los campesinos mismos. Éstas se refieren a dos campos semánticos distintos : la fisiología humana y les técnicas textiles. En este artículo proponemos explicitar dichas analogías siguiendo el

* Docteur en ethnologie, Laboratoire d'ethnologie et de sociologie comparative (UMR 7186), Maison Archéologie et Ethnologie, 21 allée de l'Université, 92023 Nanterre [ingridhallp@yahoo.fr].

Journal de la Société des Américanistes, 2012, 98-1, pp. 101-131. (C) Société des Américanistes. 
ciclo agrícola. Pero nuestro propósito no es tanto ofrecer una interpretación de las analogías, sino más bien analizar la manera como éstas se estructuran entre sí. Utilizaremos para eso el concepto de « esquema generador de la práctica » de Bourdieu (1980) y él de « esquema integrador» propuesto por Descola (2005). [Palabras claves : analogías, técnicas agrícolas, tejido, fisiología humana, concepto de esquema, Andes, Perú.]

L'observation de la mise en culture d'une parcelle irriguée a permis de relever un certain nombre de correspondances entre différents champs sémantiques : la terre et le sang sont considérés d'une façon similaire ; l'eau d'irrigation transporte le souffle d'une divinité ; les végétaux ont une âme à l'instar des êtres humains ; une parcelle est comparée à une pièce textile... Le terrain d'étude, une communauté paysanne de la région de Cusco, située dans le district de Calca (Figure 1), semblait pourtant bien peu «traditionnel». Le groupe social concerné avait été constitué suite à la réforme agraire de 1969 ; plus de la moitié des soixante familles qui le composaient était convertie au protestantisme et le quechua n'était plus la seule langue utilisée au quotidien. Malgré cela, tout au long du cycle agricole, ces paysans mettaient la culture irriguée en relation avec la physiologie humaine et la confection d'une pièce textile.

Au début de notre enquête ${ }^{1}$, nous avions travaillé sur les techniques agricoles alors que la plupart des rituels agricoles avaient, pour leur part, disparu. Les correspondances entrevues étaient donc d'autant plus fascinantes et importantes à étudier que les aspects « symboliques » semblaient a priori bien loin. On pouvait les qualifier d' « analogies » car elles semblaient dépasser la figure littéraire de la métaphore et induire une ressemblance partielle, voire plus générale, entre deux éléments (Le Petit Robert 2003, p. 88). Néanmoins, l'emploi de ce terme ne suggère pas que nous souscrivons entièrement à la théorie « analogique » défendue par Descola (2005). En effet, si nous partageons avec cet auteur le constat selon lequel certaines sociétés - dont les sociétés andines - ont abondamment recours aux analogies, nous ne pensons pas en revanche que leur usage relève d'un régime ontologique spécifique. Nous pensons plutôt, comme Bourdieu (1980) ou Lakoff et Johnson (1985), que c'est l'un des mécanismes les plus communs de l'esprit humain. Plusieurs ethnographies l'attestent d'ailleurs (voir, par exemple, Griaule 1975 ; Bourdieu 1980 ; Lévi-Strauss 1991). Ce point est important, car il permet de souligner le fait que notre propos n'est pas de démontrer une spécificité andine (et d'abonder dans le sens de lo andino, à savoir ce qui est intrinsèquement andin), mais de réfléchir sur un mode de pensée, partagé par un grand nombre de populations, qui mobilise de nombreuses analogies.

Notre approche, contrairement à ce qui se fait souvent dans les Andes pour aborder l'analyse des analogies, n'est pas à proprement parler structuraliste, nous n'aurons pas recours à une analyse structurale des mythes. Nous accorderons aux rituels, aux techniques et aux savoirs un statut équivalent à celui des mythes alors 


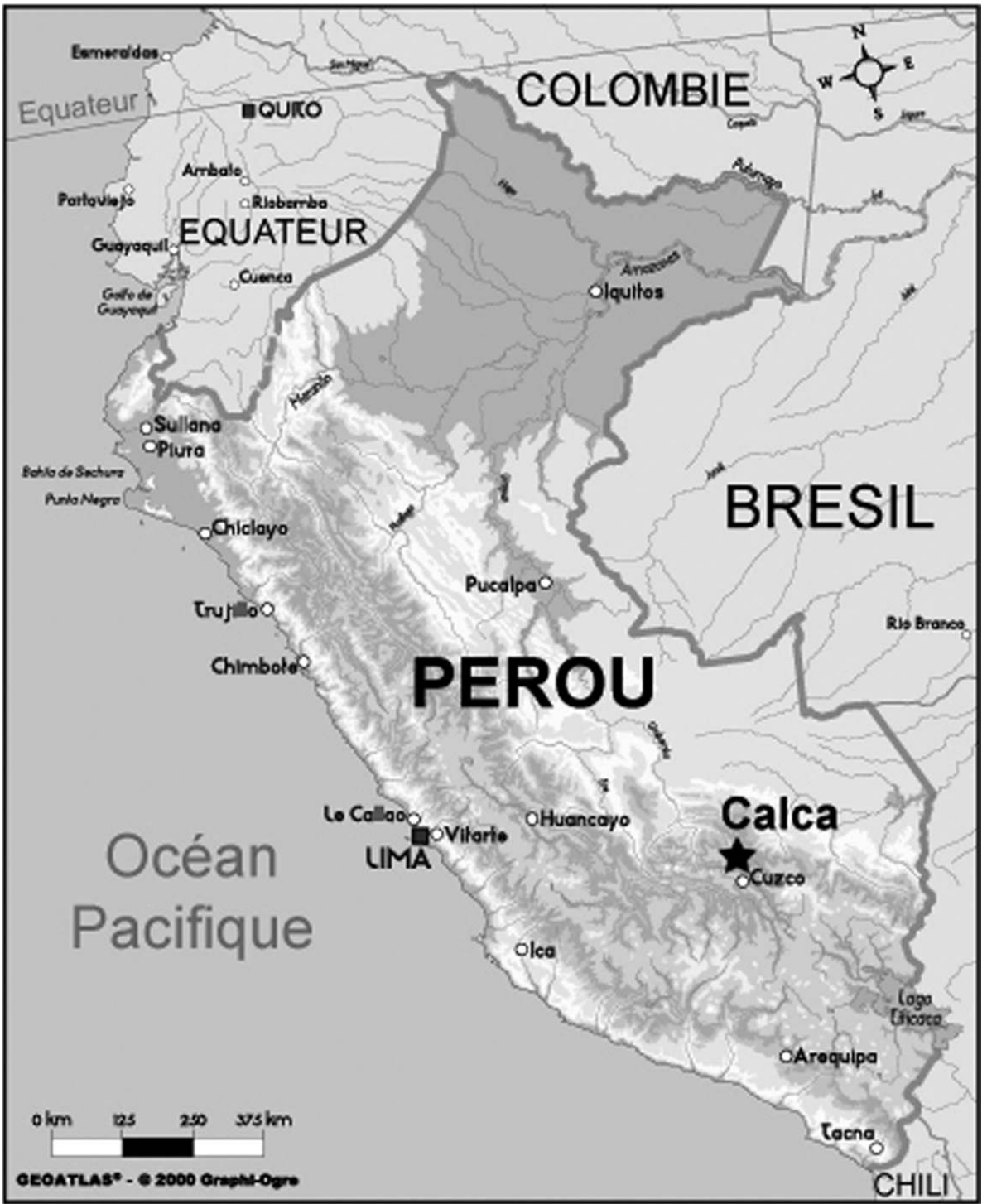

FIG. 1 - Localisation de la communauté où a été réalisé le terrain (C 2012 Geoatlas).

que, dans l'analyse structurale, ces éléments servent surtout à interpréter les matériaux mythologiques. Par ailleurs, nous ne rechercherons pas les oppositions et inversions qui fondent la méthode structurale. Ce choix a été motivé par une intuition, à savoir que, si structures mentales il y a, celles-ci sont forgées dans et 
par la pratique. L'importance de la pratique a été soulignée par un certain nombre d'auteurs, dont Haudricourt (1962), Bourdieu (1980), Descola (2005), Latour et Lemonnier (1994). Cependant, comme le constate Boyer (1993), il existe encore un certain tâtonnement conceptuel. C'est pourquoi nous avons estimé nécessaire de présenter les données ethnographiques.

Dans l'anthropologie andiniste ${ }^{2}$, les correspondances entre champs sémantiques différents abondent, mettant en parallèle une pièce textile avec un territoire communal, l'eau avec une substance corporelle, prêtant aux plantes une âme à l'instar des êtres humains etc. Ces analogies sont souvent interprétées de façon ponctuelle comme relevant d'associations symboliques. Une difficulté se pose alors car, considérées séparément, elles peuvent livrer des interprétations multiples et contradictoires: par exemple, des divinités sont masculines dans tel contexte, féminines dans tel autre (Allen 2002, p. 33). Notre objectif est de montrer que ces analogies prennent sens non pas de façon isolée, mais comme faisant partie d'un tout, et de dégager la logique qui les ordonne.

Nous présenterons, dans un premier temps, les données ethnographiques relatives à la mise en culture d'une parcelle irriguée qui permettront de rendre compte des analogies mobilisées par les paysans. Nous analyserons ensuite la façon dont ces analogies sont agencées les unes par rapport aux autres afin d'en proposer une interprétation tout en posant les jalons d'une réflexion théorique.

\section{ETHNOGRAPHIE DE LA MISE EN CULTURE D'UNE PARCELle IRRIGUÉE}

L'ethnographie que nous allons présenter ici nécessite une double mise au point. En premier lieu, toutes les terres de la communauté ne sont pas irriguées ${ }^{3}$, aussi on pourrait penser que les données exposées ne sont pas représentatives de la communauté en question. Pourtant il n'en est rien. Les entretiens menés auprès de paysans sur des terres non irriguées ne démentent pas la logique dégagée. Second point méritant d'être souligné, la plupart des rites, plus ou moins élaborés, décrits dans l'article, ont aujourd'hui disparu à Llanchu sous l'influence des « évangéliques ${ }^{4}$. Les conversions se sont tellement multipliées (plus des deux tiers de la population) au cours des dernières décennies que les catholiques eux-mêmes ne les exécutent plus que très discrètement ${ }^{5}$ et refusent généralement d'en parler. Néanmoins, les jeunes adultes les connaissent pour avoir vu leurs parents les réaliser. Aussi, à défaut de constituer un ensemble de pratiques communes, ces rites et les représentations qui les sous-tendent font partie d'un fond culturel commun qui peut être mobilisé, hors du champ rituel, par la parole et la mémoire. C'est pourquoi ils ont été inclus dans l'ethnographie. Le rôle des anciennes divinités apparaît vraisemblablement affaibli, mais peut-être était-ce là le prix à payer pour voir l'importance d'autres éléments, comme les savoirs et les techniques, autrement relégués hors champ. 
Posant l'hypothèse que les analogies sont liées à la pratique et aux techniques, nous prendrons l'ethnographie dans la perspective des chaînes opératoires. Si un point relatif à une analogie nécessite une explicitation, nous la donnerons avant de reprendre le déroulement des actions relatives à la mise en culture de la parcelle.

\section{Rite du $1^{\text {er }}$ août}

Dans la région de Cusco, si le cycle agricole commence en septembre, la nouvelle saison végétative est ouverte début août par le rite du « paiement à la terre » (esp. ${ }^{6}$ pago a la tierra) qui a lieu les tout premiers jours du mois. Malgré l'abandon des rituels, cette date continue à marquer le début de la nouvelle saison. Le rituel était une étape préparatoire aux travaux agricoles (Valderrama et Escalante 1988, p. 110 ; Marzal 1971).

Ce rite consiste, donc, en un « paiement à la terre ». Le terme pour désigner « la terre» à Llanchu est généralement tierra (esp.). Pourtant, lorsqu'il est question de rituel ou de croyances (esp. creencias), les termes de Pachatierra, Madame Juana (Doña Juana) ou Plaine Rouge (Pampa Roja) sont plus volontiers utilisés et supplantent celui de Pachamama cité dans la littérature. Des fonctions physiologiques humaines peuvent être attribuées à la terre par les paysans. Début août, la terre est considérée comme « ouverte », « à cette date, elle attrape tout parce qu'elle a faim » (Natalia, 30 ans, catholique, 2005) ${ }^{7}$. Dans la communauté de Surimana (Canas, Cusco), l'ouverture de la terre renvoie à celle du corps de la femme au moment où elle est la plus fertile, c'est-à-dire lors des règles selon les conceptions andines (La Riva 2000, p. 172). Par ailleurs, la terre peut « attraper» (esp. agarrar, que. hapiy) la force vitale des gens, les rendant malades. Le rite " pour la terre », explique Nemesio Condori (47 ans, catholique, 2005), c'est « pour qu'elle te protège, pour qu'il ne t'arrive rien [de mal], pour que la terre produise mieux, pour augmenter la production ». Concrètement, au cours de ce rituel, un spécialiste présente à la Pachatierra un despacho (esp.), un paquet d'offrandes rituelles acheté sur le marché, accompagné de feuilles de coca et d'alcool. Seul l'homme de la maison est également présent lors de cette cérémonie nocturne. Les offrandes sont adressées aux $a p u^{8}-$ les divinités ${ }^{9}$ des montagnes liées à la localité - ainsi qu'à la terre; elles seront brûlées puis enterrées. Les paysans de Llanchu, lorsqu'ils décrivent ces rituels, mettent en avant deux partenaires qu'il faut « atteindre » (esp. alcanzar) en soufflant sur les feuilles de coca, puis en brûlant les offrandes : les apu et la Terre. Dans ce contexte, les apu sont considérés comme un ensemble de divinités masculines associées aux sommets des montagnes, tandis que la Terre est une divinité féminine. Face à la multiplicité des divinités masculines, hiérarchisées entre elles, la Terre apparaît comme une entité féminine générique. Allen (2002, p. 33) signale, en se fondant sur un terrain réalisé dans la même région de Cusco 
(Paucartambo), que « alors que la terre en général est considérée comme féminine, les multiples tirakuna [apu] hiérarchisés sont considérés comme masculins ». La Terre est au centre des préoccupations des paysans. Elle impose sa temporalité, doit être " ouverte » et affamée pour accepter les offrandes. Pourtant, malgré cette place centrale accordée par les paysans à la terre dans leurs commentaires, le rôle actif des divinités masculines est également souligné, c'est à elles qu'ils s'adressent plus spécifiquement dans les oraisons.

\section{Irrigation de la parcelle}

Le cycle agricole à proprement parler commence, début septembre ou courant octobre, avec l'irrigation de la parcelle. Après la saison sèche, le sol est impénétrable. La parcelle se trouve telle qu'elle était après la récolte précédente, on voit encore les sillons en relief et les pieds des végétaux jonchent le sol. La terre, très dure, doit être irriguée pour pouvoir être labourée. Puis, pour semer, il est nécessaire d'apporter une quantité d'eau importante pour l'ameublir et la gorger d'une eau qui alimentera les plantes. Cette première irrigation permet d'avancer la date du semis et d'allonger le cycle agricole. Cette tâche incombe au chef de famille qui, armé d'une pioche, va chercher l'eau du canal et l'amène par une rigole jusqu'à la parcelle. Une étape rituelle aujourd'hui presque complètement disparue précédait systématiquement l'application de l'eau à la parcelle. Selon Roberto (41 ans, évangélique, 2005) - dont le père continue discrètement à officier -, il faut « marier (matrimoniar) l'eau avec la terre (...) en soufflant, avec de la coca, de l'alcool et de la bière de maïs (chicha) ». Il s'agit ici d'un rite plus simple que le despacho précédent, une tinkasqa que chacun est à même d'accomplir lui-même. Pour ses secondes noces, la Terre semble changer de partenaire, aux apu succède l'eau. Entre ces deux partenaires, il y a une différence de taille car l'eau n'est pas considérée comme une divinité, ni même comme une entité personnifiée. Sur ce point, catholiques et évangéliques sont d'accord et affirment ensemble que l'eau est un élément inerte (" es un elemento no más »). Néanmoins, cette eau est porteuse d'un quelque chose qui inquiète. J'ai fini par faire un lien entre les nombreuses mises en garde qui m'étaient adressées (" no toques agua, te va hacer daño ») et d'autres données. Par exemple, Lestage (1999, p. 145) explique qu'à Laraos, " les femmes sont plus souvent victimes de l'eau que les hommes ». La crainte qui s'exprime le plus facilement à Llanchu concerne l'irrigation : les femmes sont réticentes à l'idée d'irriguer. Les catholiques évoquent la présence de crapauds qui pourraient les attaquer attirés par leurs menstrues, tandis que les « évangéliques » parlent plus volontiers du risque d'encourir une inflammation des ovaires. Dans les deux cas, les craintes des femmes portent sur leurs organes reproducteurs et, donc, leurs capacités génésiques.

D’autres enquêtes ethnographiques réalisées à Llanchu ont montré des pathologies spécifiques liées à l'eau. La maladie du pukyo, terme quechua 
désignant aussi bien une résurgence qu'une maladie, consiste en une affection des membres (les jambes se tordent ou arrêtent leur croissance - esp. se encogen), en des gonflements ou des affections de la peau. La première de ces affections pourrait correspondre à la poliomyélite. Les paysans interrogés précisent que c'est un apu qui se manifeste, à travers la source, mangeant un os ou insufflant un principe aqueux. Fortunato Huaman (61 ans, catholique, 2004) est tombé malade alors qu'il travaillait avec d'autres ouvriers à l'aménagement d'une résurgence d'eau chaude. Pendant un mois, alors qu'ils entraient et sortaient de l'eau, ils n'avaient eu aucun problème. Puis un jour, le genou de Fortunato s'était mis à gonfler et un liquide jaune s'en écoulait continuellement. Les différents traitements entrepris ne donnèrent aucun résultat jusqu'à ce qu'un guérisseur diagnostique, finalement, un pukyo. Ce dernier fit des offrandes à un apu soigneusement identifié et Fortunato guérit. Quand j'ai demandé à Fortunato comment il expliquait qu'ils ne soient pas tous tombés malades dès le premier bain, il m'a expliqué que l'apu qui se manifestait dans cette eau avait respiré juste quand il était dans l'eau, il avait alors " attrapé » son animu (esp./que.) - énergie vitale dont il avait gardé une partie. Autrement dit, il s'était trouvé au mauvais endroit au mauvais moment. C'est l'« haleine » (esp. aliento) de la divinité qui l'avait affecté et c'était donc à cette divinité qu'il fallait faire une offrande, ce que le ritualiste avait fait. De façon générale, les dangers liés à l'eau sont associés aux émanations gazeuses, aux bulles d'air qui affleurent dans de nombreuses sources. Pour les paysans, ce sont autant de preuves que l'apu respire. L'eau des sources est conçue comme un fluide qui transporte de l'énergie vitale en provenance de la divinité, à l'instar du sang des hommes qui véhicule leur animu (La Riva 2005). L'animu des hommes est volatile, comparable à l'haleine (que. samay; esp. aliento) et souvent comparé au vent (que. wayra; esp. viento) (ibid., pp. 65-66, p. 74). L'association entre animu et haleine est si forte qu'un interlocuteur évangélique m'a expliqué que seuls les humains et les animaux ont un animu, la respiration étant la preuve de la présence d'une âme. Il apparaît donc que les paysans de Llanchu conçoivent la physiologie de l'apu sur le modèle de la physiologie humaine. L'eau d'irrigation semble, à l'instar de l'eau de source, être assimilée à un fluide physiologique, masculin en l'occurrence.

Juste après ce rite de la tinkasqa, dont la durée et le degré de formalisation ne sont pas en rapport avec la quantité d'explications ethnologiques qu'il suscite, le paysan commence à irriguer sa parcelle. La première irrigation est exclusivement réalisée par des hommes. La technique utilisée est appelée « irrigation par inondation » car la quantité d'eau est plus importante que pour les autres irrigations. En effet, la terre est desséchée, très dure et il faut beaucoup d'eau pour bien l'imbiber et pouvoir la travailler. Pour irriguer, l'homme s'arme d'une pioche dont la pointe sert à creuser des rigoles. L'eau du canal est ainsi guidée dans de multiples rigoles creusées dans le sens de la pente. Une certaine quantité d'eau s'écoule dans chaque sillon perpendiculairement à la pente et, de proche en 
proche, la rigole humecte un ruban de terre. L'érosion du sol, plus prononcée en haut, permet d'aplanir les terrasses (en formation sur la Figure 2).

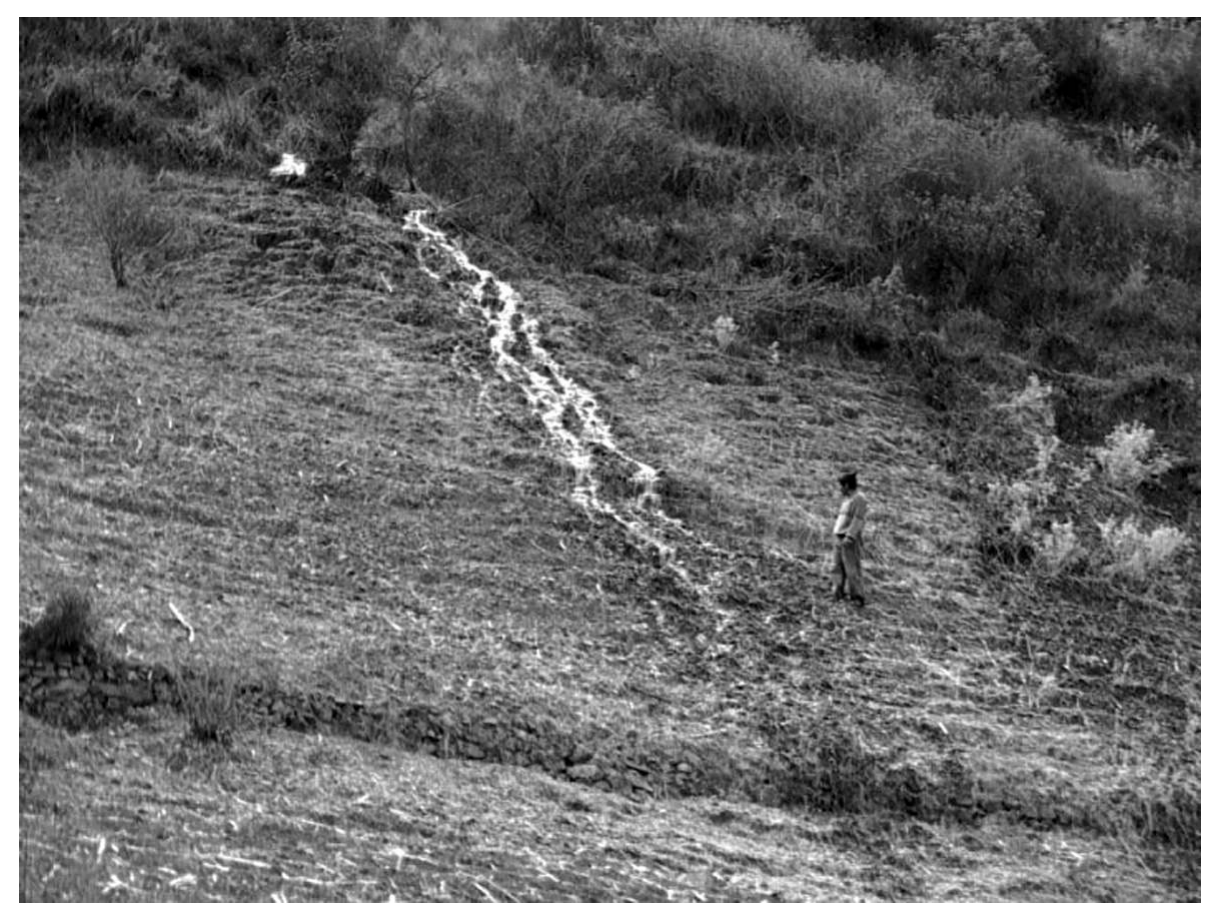

FIG. 2 - Première irrigation (cliché Hall).

La quantité d'eau lors de cette irrigation dépend du type de sol. Selon la proportion d'argile dans le sol, les paysans expliquent que la terre «supporte » (esp. aguanta) plus ou moins bien l'apport massif d'eau. Pour pouvoir ensemencer une parcelle, il importe en effet que la terre soit bien humide, mais elle ne doit pas se transformer en « boue » (esp. barro). Les hommes estiment être les seuls à pouvoir évaluer la nature du sol, critère indispensable pour bien irriguer.

En étudiant la classification des sols, il est apparu que cette dernière coïncidait point par point avec celle du sang. À la question « de quel type de sol s'agit-il ?» (esp. ¿Qué clase de tierra es ésta?), les paysans de Llanchu répondent presque exclusivement en mentionnant la couleur de la terre et utilisent plus facilement le quechua pour énoncer les trois couleurs de terre qu'ils distinguent : noire (que. yana), rouge (que. puka) et jaune (que. $\left.q^{\prime} i l l u\right){ }^{10}$. Dans la pratique, s'il est facile d'identifier la terre jaune, en revanche, différencier un sol rouge d'un noir est loin d'être évident. Plus qu'à des couleurs bien distinctes, les termes utilisés renvoient 
à des nuances d'une même couleur. La terre noire est la plus commune et, également, la plus appréciée. C'est la terre la plus « forte » (que. kallpayuq), celle qui produit les meilleures pommes de terre. Il n'est nul besoin d'ajouter du fumier, de l'engrais ou des pesticides, et non seulement cette terre est « forte », mais elle est également « saine ». Généralement situées au-dessus de 4000 m d'altitude, les parcelles de terre noire sont cultivées pendant deux ans, puis laissées en friche quatre à cinq ans. Du point de vue agronomique, les conditions climatiques et les longues périodes de repos auxquelles ces sols sont soumis permettent à la terre d'être riche en matière organique, ce qui lui donne cette couleur sombre. Les paysans insistent sur la résistance de ces terres face au manque ou à l'excès d'eau : ils disent qu'elles " supportent » (esp. aguantan) bien ces variations. Quel que soit leur degré d'humidité, ces sols gardent une structure très fine et la matière organique permet de modérer les effets de la forte proportion d'argile. À des altitudes inférieures, entre $3800 \mathrm{~m}$ et $3300 \mathrm{~m}$, le taux de matière organique diminue car la température augmente et le temps de repos est écourté. En conséquence, la terre est moins sombre en surface et devient rouge, au moins en profondeur. Pour les paysans, ces terres rouges ne sont pas d'aussi bonne qualité que les noires : elles sont moins «fortes » et il est nécessaire d'utiliser un engrais pour les cultiver. Les paysans « renforcent» ces terres en leur apportant du fumier et du guano ou, encore, en ajoutant de la terre noire. Les parcelles de terre rouge sont plus sensibles aux parasites et autres maladies, aussi l'usage de pesticides est très fréquent. Ces sols rouges sont donc doublement faibles, ils sont d'ailleurs qualifiés de "couards » (esp. cobardes). Pour justifier l'emploi de ce terme, les paysans font référence au comportement de ces sols en fonction du climat : ils se craquèlent en cas de sècheresse et deviennent boueux après une forte pluie. Contrairement aux sols noirs, "ils ne supportent pas bien " (esp. no aguantan) les variations d'humidité. Les paysans considèrent qu'en cultivant ces parcelles, leurs qualités s'améliorent et ils constatent qu'un sol rouge cultivé noircit (esp. ennegrece) avec le temps. De fait, la quantité de matière organique d'un sol cultivé augmente avec le temps, aussi devient-il plus sombre. La dernière catégorie de terre est de couleur jaune. Sur ce type de sol, très rare, rien ne pousse. Il s'agit d'argile presque pure qui ne sert qu'à préparer un enduit appliqué sur les murs de terre crue ou sur le foyer de la cuisine. Pour cela, cette terre est idéalement mélangée avec un peu de terre rouge et de terre noire.

Cette classification repose donc sur un critère chromatique qui permet d'évaluer globalement la qualité d'un sol. Plus que de couleurs différentes, il s'agit en fait de nuances qui, comparées entre elles, permettent d'élaborer une classification et une hiérarchie. Les nuances les plus sombres sont les plus fortes. Par «force », il faut entendre plusieurs aspects comme la fertilité, la richesse en matière organique et en argile, la résistance aux maladies et aux parasites et le comportement face aux variations d'humidité. Par ailleurs, il apparaît que l'appréciation en termes pédologiques des sols se teinte de valeurs 
morales. En effet, par exemple en ce qui concerne le comportement des sols face à l'excès ou au manque d'eau, tandis que les uns sont considérés comme valeureux et supportant tout, les autres sont perçus comme couards et ne sachant pas endiguer les excès.

Penchons-nous à présent sur la classification du sang. Les paysans de Llanchu, à l'instar de ce que nous avons vu pour les sols, distinguent trois types de sang par leur couleur : noir, rouge et jaune ${ }^{11}$. Les classes de sangs noir et rouge sont souvent les deux seules évoquées spontanément lors des entretiens avec les paysans. Comme dans le cas des sols, mais de façon plus évidente encore, la classe jaune fait l'objet d'un traitement à part. Le sang jaune n'est évoqué que pour parler de celui des malades sur le point de mourir, leur sang n'étant pas viable. Les personnes dont le sang est noir ou rouge sont, elles, sinon en bonne santé, du moins pas à l'article de la mort. D'après les paysans, le noir est plus « fort » que le rouge, à tel point que le premier serait « positif » et le second « négatif » (Roberto Huallpa 41 ans, évangélique, 2004). La force du sang donne une idée de l'état de santé d'un individu, mais renvoie également à son caractère et à son statut social. En effet, les hommes respectés ayant acquis un statut social élevé par les services rendus à la communauté sont censés avoir un sang noir. La maturité individuelle et sociale s'accompagne de l'assombrissement de la couleur du sang. Dans les communautés du district tout proche de Pisac, Pérez Galán (2004) a indiqué que « passer des charges » se dit « faire son sillon » (que. wachu). Il y aurait donc un lien entre le fait de monter dans la hiérarchie sociale et de cultiver une terre : ces deux actions ont comme conséquence d'assombrir le sang ou la terre et de les renforcer. En contrepoint, avoir le sang rouge indique une certaine fragilité, de caractère ou physique. Par ailleurs, la couleur du sang dépend également du sexe de l'individu, car les femmes fertiles sont réputées avoir le sang rouge. Si avoir le sang rouge n'est pas pathologique, en revanche les nuances plus claires indiquent une dégradation de l'état de santé, tant pour les hommes que pour les femmes. La perte de couleur indique une diminution de l'énergie vitale contre laquelle des mesures thérapeutiques doivent être prises. De façon générique, les faiblesses physiques sont appelées animia à Llanchu et sont censées être accompagnées d'un éclaircissement du sang. Il s'agirait non pas d'anémie - esp. anemia -, comme on pourrait le penser, mais d'une affection de l'animu, de l'énergie vitale ${ }^{12}$. Ce principe vital englobe la force physique (que. kallpa) pour la dépasser.

Ainsi, les classifications des sols et des sangs, toutes deux fondées sur le même critère chromatique, font apparaître trois catégories hiérarchisées en fonction du degré de force accordé à chacune. De plus, il est apparu que le fait de cultiver (que ce soit de façon agricole ou sociale au sens d'apprentissage des usages sociaux) rend le sol ou le sang plus sombre, donc plus fort. À partir de tous ces éléments, il est possible d'en déduire une homologie classificatoire entre le sang et le sol. Reste à savoir si cela permet de supposer que les paysans associent effectivement la terre et le sang ${ }^{13}$. 
Tandis que le rite du $1^{\mathrm{er}}$ août viendrait sceller l'union entre la Pachatierra et l'apu, l'étape suivante ne marquerait-elle pas la rencontre des « fluides corporels » des divinités déjà mariées ? En effet, du côté masculin, il apparaît très nettement dans les entretiens que l'eau est conçue comme une substance physiologique de l'apu, la divinité masculine ${ }^{14}$. Du côté féminin, la terre renvoie, par analogie, au sang. Grâce à ce changement de perspective, les partenaires rituels sont de nature comparable. Ainsi, la terre du premier rite serait la divinité Pachatierra, tandis qu'il s'agirait de son sang dans le second. Cette hypothèse prend une dimension toute particulière lorsque l'on tient compte des représentations liées à la conception humaine dans les Andes : l'embryon humain résulte de l'entrée en contact du sperme et du sang menstruel (La Riva 2000, p. 171 ; Platt 2002, p. 8). Selon ces mêmes auteurs, le sang menstruel coagulerait sous l'action du sperme et ce caillot deviendrait embryon, puis fœtus, en se nourrissant du sang maternel (puisque le sang menstruel ne s'écoule plus). Le fœtus serait donc constitué de sang, ce qui fait dire à Platt (ibid.) que la conception humaine prend le «langage du sang ». Ainsi, les substances de deux partenaires, qu'ils soient humains ou végétaux, mais de sexes opposés doivent entrer en contact pour qu'il y ait fécondation. Supposer qu'il existe une identification analogique entre la terre et le sang s'avère donc très fructueux et ouvre de nouvelles perspectives.

\section{Préparation du sol}

Après la première irrigation, la terre est très humide et il faut la laisser sécher (esp. secar) quelques jours pour que l'eau pénètre bien en profondeur. C'est seulement ensuite que les labours pourront commencer. Le travail se fait collectivement au sein de groupes d'entraide selon une modalité appelée ayni (que.), une prestation réciproque et symétrique : chaque journée de travail donnée devra être rendue. Les équipes réunies comptent une petite dizaine de personnes, souvent des frères, des cousins et des beaux-frères. Bien souvent, la date qui aura été retenue de façon collective, en fonction des contraintes des uns et des autres, permettra de déterminer celle de l'irrigation. La journée est ponctuée de pauses au cours desquelles bière de maïs et aliments préparés par la famille propriétaire de la parcelle sont offerts. Cette occasion est très importante : il est essentiel de bien recevoir, le dernier repas de la journée est donc particulièrement soigné.

La journée de travail commence par les labours. Sous l'action de l'araire tiré par deux taureaux, les mottes de terre sont fragmentées. Le nombre de passages dépend de l'état du sol, la terre doit être suffisamment meuble, bien travaillée (que. allin), pour que les plantes puissent germer et croître facilement. Cette phase préalable est essentielle et s'accompagne d'un nettoyage de la parcelle. Derrière l'araire, à chaque passage, les hommes présents récoltent les pommes de terres « natives » (esp. nativas), enlèvent les pieds desséchés de maïs, nettoient les bords de la parcelle. C'est de cette manière que la terre est rendue bien meuble. 
Les paysans utilisent couramment deux qualificatifs pour décrire la texture idéale à obtenir : elle doit être « farineuse » (esp. harinosa; que. haq'o) ou encore « bien serrée» (esp. bien túpida). Ces termes renvoient à des associations sémantiques très riches et très différentes. Le qualificatif « farineux » est fréquemment utilisé pour parler du jaune d'œuf ou de certaines pommes de terre, quand l'un et l'autre sont cuits. Plus les pommes de terre sont farineuses, plus elles sont valorisées : la variété peruanita ${ }^{15}$ est particulièrement appréciée ; elle est comparée par sa texture et la couleur de sa chair à des jaunes d'œufs cuits. Ces aliments ont deux points communs : ils crissent, d'une certaine façon, sous la dent et sont tous deux des embryons. Le jaune d'œuf donnera le poussin, la pomme de terre une plante ${ }^{16}$. Cereceda (1987) s'est intéressée à la texture farineuse à travers le champ sémantique de la beauté dans les Andes. Cet auteur (ibid., p. 224) émet l'hypothèse que cette texture a été sélectionnée par la pensée mythique comme une situation limite. L'appréciation "farineuse » renvoie également au champ sémantique du textile. Une terre «bien serrée » est une terre finement travaillée, bien retournée. De façon tout à fait intuitive, la laine nettoyée et démêlée manuellement avant d'être filée a une texture floconneuse que l'adjectif « farineux » pourrait traduire, tandis que le fil, finalement obtenu, devra être fin, régulier et solide pour être filé « bien serré ».

À explorer les champs sémantiques liés à la texture idéale de la terre labourée, il apparaît que les labours pourraient être mis en parallèle avec la préparation d'un fil de laine. Si les données boliviennes sont claires, celles provenant du Pérou sont plus floues ${ }^{17}$. Les analyses fragiles que nous venons d'esquisser reçoivent une certaine validation à l'étude de l'étape suivante.

\section{Le semis}

\section{Les deux derniers labours}

Pour être semée, quelques jours après la préparation du sol, la terre devra être labourée une nouvelle fois. Appelés respectivement "premier labour» (esp. primera reja) et « second labour » (esp. segunda reja), l'avant-dernier et le dernier passage de l'araire sont singularisés. Cette terminologie est utilisée même s'il y a plus de deux labours (ce qui arrive notamment si celui qui cultive le champ n'a pas de taureaux pour labourer, seul et à l'avance, sa parcelle). Pour la clarté du propos, je m'en tiendrai au «premier» et au «second » labours. D'un point de vue technique, ils n'ont rien de particulier par rapport aux précédents. Après les quelques jours de repos, la couche superficielle peut être un peu sèche ; ces labours permettent de finir d'ameublir la terre et de s'assurer que le degré d'humidité est bien homogène. Les paysans qui dirigent l'araire effectuent, à chaque passage, des allers et retours parallèles décalés d'une trentaine de centimètres à chaque demi-tour. Cela demande aux laboureurs qui doivent guider fermement les taureaux une certaine dextérité. Le second labour sera perpendiculaire au pre- 
mier et il sera parallèle aux courbes de niveau. Celui-ci permet de former les raies dans lesquelles les graines de maïs ou les petites pommes de terre seront semées, de créer les futurs sillons de la parcelle entre lesquels l'eau s'écoulera lentement. Sur la Figure 3, on voit l'araire qui passe pour la segunda reja et, derrière, les hommes sèment le maïs, puis ajoutent les divers engrais.

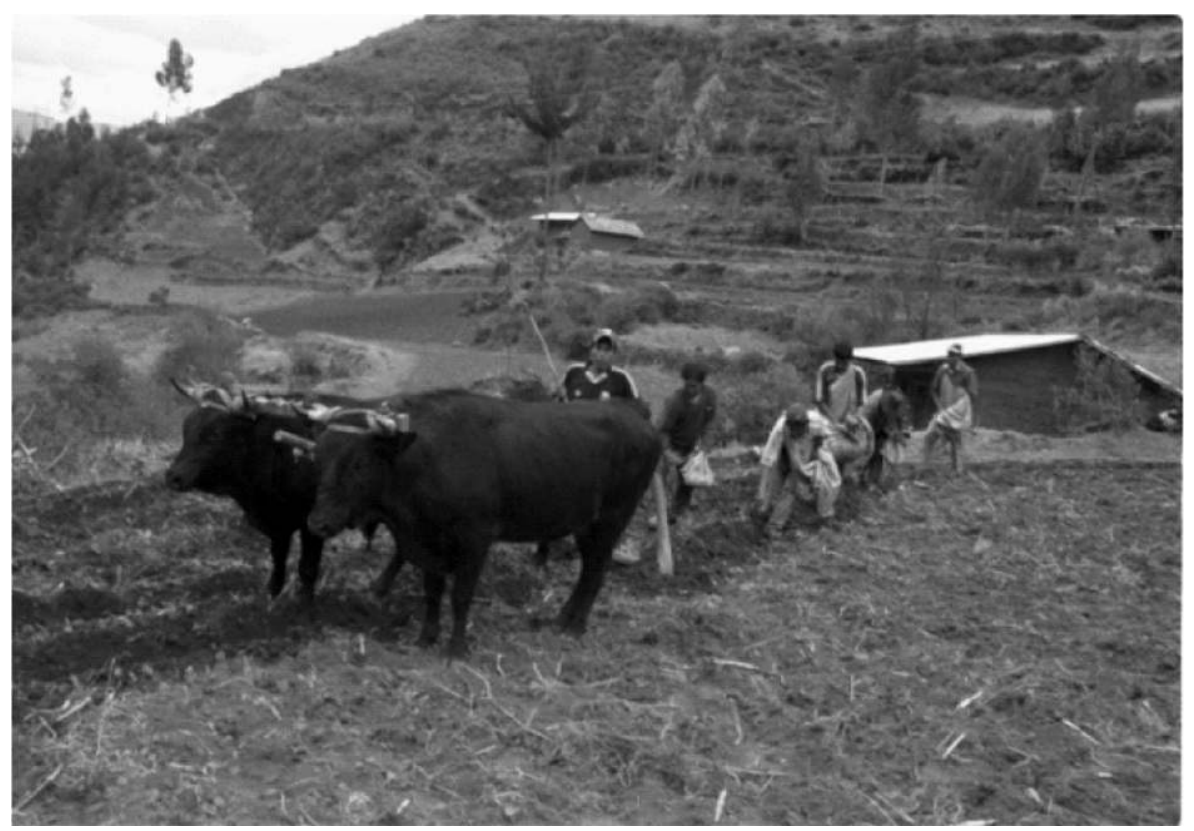

FIG. 3 - Dernier labour ou segunda reja (cliché Hall).

À Llanchu, ces derniers labours sont pensés à travers une analogie textile : les mouvements du laboureur sont formellement mis en relation avec ceux de la tisserande. Rosa (43 ans, évangélique, 2005) exprime sans hésiter comment « l'homme tisse en labourant dans un sens, puis dans l'autre », tandis que son mari (41 ans, évangélique, 2005) affirme au cours d'un autre entretien que « les hommes tissent [...], c'est comme ça toujours, on dit qu'ils tissent, toujours et tous. Les femmes sèment les motifs sur le tissu, c'est comme la parcelle ». Outre l'analogie entre les gestes techniques, les interlocuteurs expriment aussi une répartition idéale des tâches : la femme tisse pendant que l'homme laboure (ce qui n'est pas propre aux Andes, il en va de même dans la Bible par exemple). Lors du " premier labour », l'homme ourdit la parcelle, chaque aller-retour revient à lancer la pelote de laine d'un côté à l'autre du métier pour former la chaîne sur le métier à tisser. Durant le " second labour », la lame de l'araire tient lieu de navette qui fait des allersretours d'un bord à l'autre du tissu pour tisser la chaîne ${ }^{18}$ (Figure 4). 


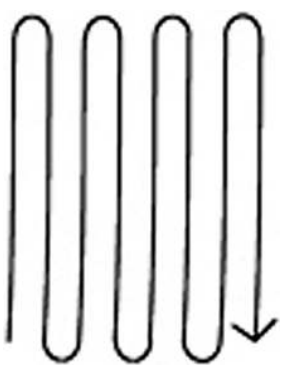

Premier labour

... ou l'ourdissage de la parcelle

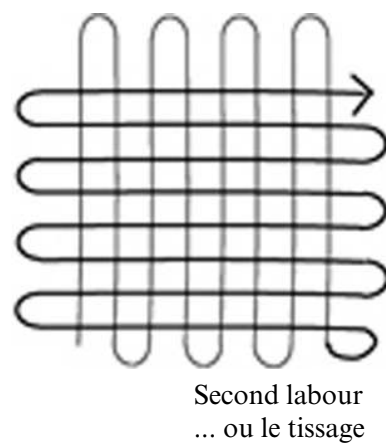

... ou le tissage

FIG. 4 - Les labours et le tissage (dessin Hall).

L'analogie entre les labours et le tissage repose essentiellement sur des similitudes techniques : les gestes décrivent un chemin identique dans leurs espaces d'application respectifs (la parcelle dans un cas, le métier à tisser dans l'autre). Le travail des bordures, qui vient finaliser l'ensemencement de la parcelle, est également mis en parallèle avec celui des lisières des textiles. Ces différentes bordures font, en effet, l'objet d'un soin tout particulier. Tandis que la majorité des hommes finissent de labourer la parcelle, la nettoient ou l'ensemencent, un ou deux jeunes hommes piochent énergiquement les bords enherbés. La terre bien ameublie est ensuite labourée et trois sillons circulaires viennent entourer les sillons centraux (Figure 5). De cette façon, toute la surface de la parcelle est utilisée. Cet aménagement périphérique s'appelle la chakapa (que.) et, pour Rosa (43 ans, évangélique, 2005), c'est un élément essentiel de l'aménagement de la parcelle : « la chakapa c'est comme une chakra (parcelle) ». Les parcelles irriguées sont majoritairement bordées par une chakapa. Certains jeunes, cependant, estiment que ce n'est pas absolument nécessaire, ils remettent en cause l'utilité technique de cet aménagement qu'ils associent à une « coutume » (esp. costumbre) de leurs parents et grands-parents. Cela les oblige à prolonger les sillons manuellement pour ensemencer toute la surface arable. Les bords des textiles font également l'objet d'une attention particulière dans les Andes où les tissus (ceux tissés par les femmes sur des métiers à tisser traditionnels) ont la particularité d'être « à quatre lisières » (Desrosiers 2000, p. 118). Il n'y a aucun nœud, le métier à tisser est retourné à la fin du tissage de telle sorte que le quatrième bord est également tissé. Les femmes terminent de tisser au cœur de la pièce de tissu et elles renforceront les bordures en les brodant ou en ajoutant des franges ou un galon de protection ${ }^{19}$.

La logique analogique se décline également d'un point de vue structurel : la parcelle tout comme la pièce de textile est organisée en bandes parallèles et aux sillons correspondent les bandes de motifs. Les décors des textiles sont réalisés grâce aux fils de chaîne de différentes couleurs et sont composés de bandes de part et d'autre de l'axe central. Ces bandes sont plus ou moins larges : de fines bandes 


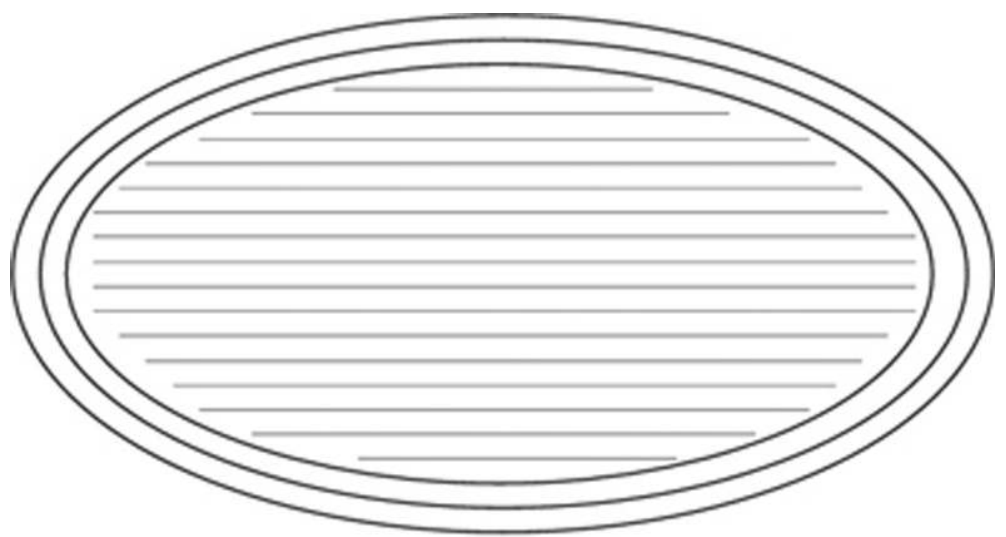

FIG. 5 - La chakapa est formée de trois sillons autour de la parcelle (dessin Hall).

monochromes de différentes couleurs alternent avec d'autres où figurent des motifs et, éventuellement, avec de larges bandes monochromes appelées «plaines » (que. pampa). De nombreux textiles, à Llanchu, ne comportent que de fines bandes de couleur (esp. listas). Les textiles (que. phallay) portent des motifs du même nom qui sont fort appréciés et qui demandent à la tisserande une certaine dextérité. Le poncho tissé par Rosa est un ley phallay (que.), sur lequel dominent des motifs floraux (Figure 6).

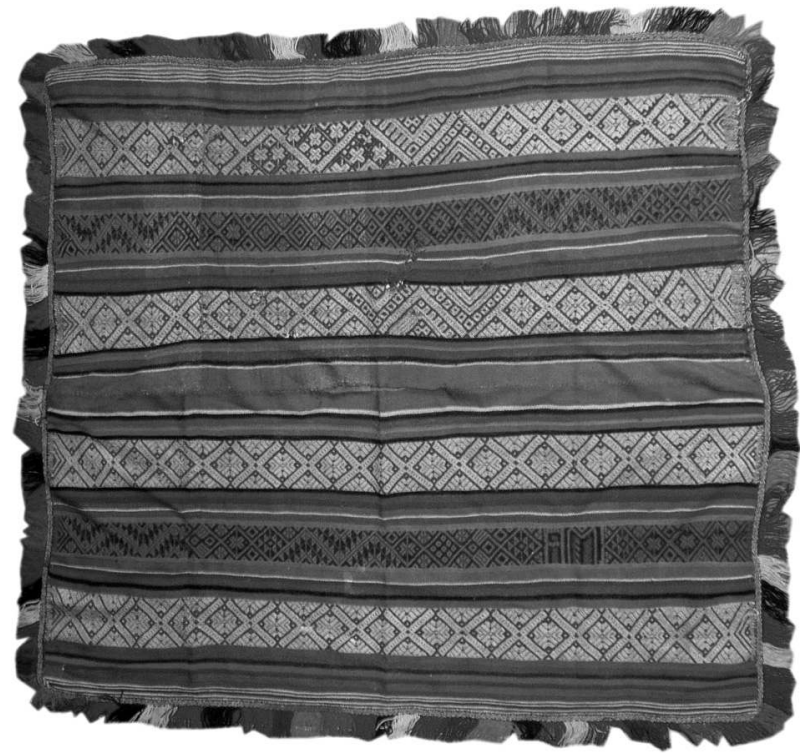

FIG. 6 - Poncho ley phallay tissé par Rosa, Llanchu (cliché Hall). 
Des motifs végétaux, comme des graines, des épis de maïs..., sont souvent représentés sur les textiles andins (Seibold 1992). Les bandes de motifs sont visiblement apparentées aux sillons de la parcelle. Une scène agricole tissée sur un sac (que. ch'uspa) bolivien vient illustrer cet aspect (Figure 7) ${ }^{20}$ : si l'on regarde le motif tissé depuis l'ouverture du sac, on voit un araire tiré par deux taureaux qui trace un sillon dans le sens de la chaîne, suis un couple de personnages dont l'un dirige les animaux et l'autre sème.

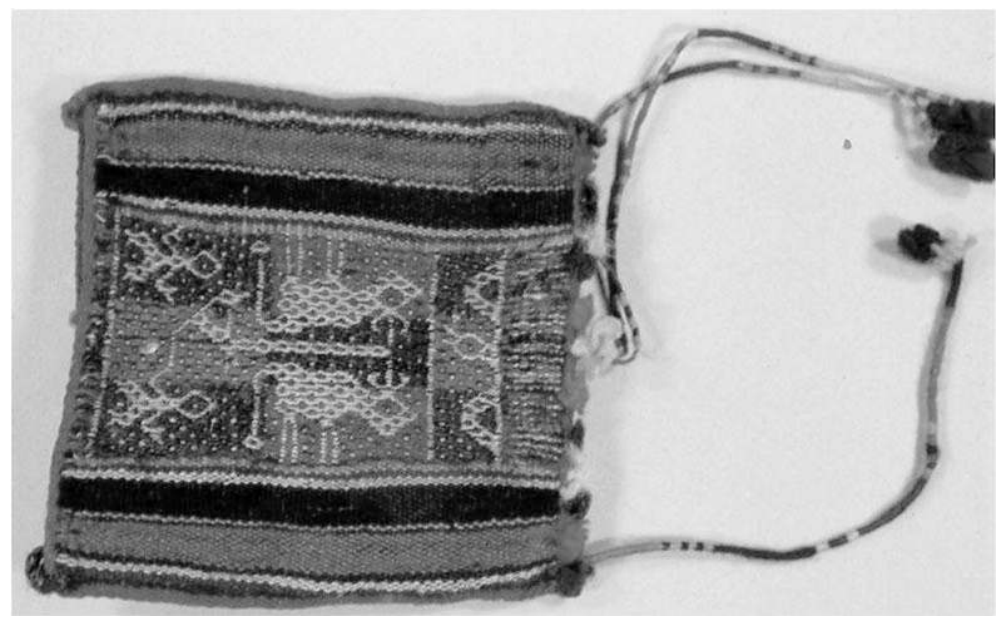

Fig. 7 - Sac (chuspa) sans doute tissé dans la région Nord-Potosí (cliché Desrosiers).

Un autre élément structurel caractéristique des textiles se retrouve dans le domaine agricole : la partition en moitiés. De nombreuses pièces textiles, comme les ponchos, les lliklla (que.) ${ }^{21}$, les couvertures ou, encore, les manta (esp.) ${ }^{22}$, sont constituées de deux laizes identiques cousues ensemble le long de leur lisière ${ }^{23}$. Dans le domaine agricole, les parcelles sont souvent divisées en plusieurs parties, appelées « moitiés » (esp. mitades), par une rigole qui permet, selon les paysans, d'obtenir des sillons d'une longueur raisonnable. Lors du sarclage, chaque homme sarcle un sillon de bout en bout. Si celui-ci est trop long, il se fatigue vite. Les paysans expliquent que c'est pour cette raison qu'ils réduisent la longueur en divisant la parcelle. De plus, cela facilite l'irrigation. D'après certains plus jeunes, cette pratique relève des coutumes des anciens, tout comme la chakapa. Remarquons que, dans la terminologie agricole, les rigoles dans la parcelle sont appelées killan (que.) qui signifie littéralement « sa lune ». À la question « De qui est-ce la lune ? », Alberto (47 ans, évangélique, 2005) répond qu'il s'agit de celle de la parcelle. Lorsque je faisais observer à Alberto que sa parcelle était divisée en trois « moitiés » et que, du coup, cela faisait, non pas un, 
mais un et demi, il me rétorquait que la lune apparaissait partiellement, mais était une. Alberto soulignait de cette façon la primauté accordée au tout sur la partie. La parcelle, à l'instar de la lune dont on ne voit souvent qu'une partie, forme un tout, une unité ${ }^{24}$.

\section{Le semis à proprement parler}

Les grains de maïs ou les semences de pommes de terre sont placés dans la raie formée par le dernier labour. Le semis commence avec un rite discret, sommaire et domestique, une tinkasqa, comme celle qui a lieu pour la première irrigation. Il s'agit encore une fois d'un mariage (le troisième ?) dont les deux partenaires sont la terre et les semences. Le propriétaire de la parcelle souffle sur de la coca en s'adressant aux apu et verse quelques gouttes d'alcool sur le sol. La terre est encore au cœur de l'action rituelle et change une fois de plus de partenaire. Comme nous pouvons supposer que la terre reste féminine, la semence doit être masculine. La terminologie soutient une telle supposition, le terme quechua muqu désigne à la fois la graine et le sperme (La Riva 2000, p. 171 ; Platt 2002). Une fois encore, on constate une analogie entre reproduction végétale et humaine. Cette analogie se heurte pourtant à une difficulté que Roberto (41 ans, évangélique, 2005) formule de la façon suivante : « s'il n'y avait pas de graine, elle pousserait où dans ce cas? C'est pas vrai ? Si tu ne mets pas la graine dans la terre ça ne pousse pas... Si tu mets les deux, ça pousse » (" i Si no habría semilla donde crecería también pé ? ¿ No cierto ?... ¿ Si no pones la semilla a la tierra, no crece... Si pones los dos, crece... »). La terre ne donnerait rien si elle ne recevait que l'eau d'irrigation. Il semble dès lors logique de célébrer un rite au moment de déposer les graines en terre, comme cela avait été le cas au moment de la rencontre des substances masculine et féminine. Par ce rite, la graine peut être considérée comme le partenaire masculin de la terre, celui avec lequel elle se marie.

Le partenaire féminin sollicité dans le rite est la parcelle, appelée par son nom, et non pas la divinité féminine générique, la Pachatierra. Quelques considérations sur les toponymes s'avèrent fort éclairantes pour comprendre le rite précédemment décrit. Il existe de très nombreux noms de lieux et à un lieu peuvent correspondre plusieurs toponymes. Des secteurs de quelques hectares ayant chacun un nom servent de référence au quotidien. Des toponymes précis existent également pour chaque parcelle, mais, en dehors des rites, ils ne sont que rarement mentionnés. Seule la famille qui exploite une parcelle connaît son toponyme. Un changement de propriétaire semble entraîner systématiquement l'attribution d'un nouveau nom. Selon Roberto (41 ans, évangélique, 2005), le nom d'une parcelle est une information précieuse qui pourrait être utilisée à mauvais escient, ce nom pourrait en effet servir à lancer un mauvais sort sur la parcelle. Ainsi, le toponyme permettrait d'agir sur la parcelle, pour « ceux qui croient en la terre », c'est-à-dire les catholiques selon la terminologie locale. Chaque famille entretient une relation privilégiée avec la parcelle qu'elle cultive. Cette manière de 
singulariser des parcelles peut être rapprochée de pratiques observées chez les Macha de Bolivie. Platt (2002) rend compte de l'existence de wirjinas (dérivé de l'espagnol vírgenes, vierges), divinités localisées dans les parcelles proches de la maison. Les parcelles seraient des entités féminines ayant chacune leur nom, tenu secret. La possibilité d'agir sur une parcelle grâce à la connaissance de son toponyme rappelle clairement le fait que le nom d'un individu contient une partie de son énergie vitale (que. animu). Comme le note La Riva (2005, p. 76), le nom est au centre de la thérapie appelée « rappel de l'âme » visant à réincorporer l'animu dans le corps d'un malade. Le nom de la personne est répété trois fois et cet acte a une dimension performative. De la même façon que connaître le nom d'un individu donne prise sur son animu, connaître le toponyme d'une parcelle permettrait d'influencer la récolte à venir. Dans le cas péruvien également, la Pachatierra serait donc une entité générique qui engloberait des entités localisées ${ }^{25}$. Lors du semis, tout se passerait comme si chaque famille mariait la parcelle qu'elle cultive avec les graines qu'elle sème, créant de cette façon une relation personnelle entre ladite famille et la portion de terre exploitée.

Reprenons le suivi de la chaîne opératoire. Le semis de la parcelle commence effectivement après le rituel. Les grains de maïs ou les petits tubercules sont déposés dans les raies creusées au cours du dernier passage de l'araire. Est ajouté ensuite du fumier d'ovins, de cochons d'Inde et d'oiseaux (ou guano). Un dernier passage avec l'araire permet d'enfouir les graines. Enfin le sol est aplani à l'aide d'une planche traînée sur le sol par les taureaux. Recouvertes, les graines peuvent alors germer et se développer. La terre doit être suffisamment humide pour ne pas devoir être irriguée pendant presque un mois.

Pour poursuivre l'analogie entre reproduction humaine et végétale, signalons que les végétaux, tout comme les embryons, possèdent une énergie vitale, un animu. Néanmoins, à la différence des êtres humains qui ont tous un animu, seuls certains plants en sont dotés (ils sont appelés taqe que.). Que ce soit pour le maïs, les pommes de terre ou les autres végétaux, ces taqe ont trois têtes, trois bourgeons apicaux qui les font ressembler de façon plus ou moins fidèle à des croix. Selon Evangelina (54 ans, catholique, 2005), ces végétaux, à la forme si particulière, sont des manifestations de l'animu de l'espèce végétale correspondante, qui est d'ailleurs dotée d'un nom. Ainsi, les végétaux sont dotés d'un animu qui se manifeste rarement, un animu générique en quelque sorte.

\section{Croissance des plantes, sarclage, irrigations ultérieures}

Dans les journées et semaines qui suivent le semis, la parcelle est laissée telle quelle, les graines germent, les racines et la tige se développent jusqu'à sortir de terre. Après un mois, les plants atteignent une hauteur d'une dizaine de centimètres. Un premier sarclage est alors réalisé (esp. primer aporque; que. hallmay). Avec des pioches (esp. pico; que. kuti), les hommes se placent chacun sur un sillon 
et sarclent la terre entre les plants de façon à creuser des raies et à reformer des sillons. Cela permet d'arracher des mauvaises herbes entre les plants, d'enfouir les autres et de préparer l'irrigation ultérieure. Ce travail se fait en équipe, au cours d'une journée d'entraide. Cette étape du travail agricole peut faire l'objet d'une compétition entre hommes, notamment lorsqu'elle est réalisée sur les terres communales : c'est à qui finira son sillon le plus rapidement. Avec la disparition des terres communales irriguées, cette pratique a cessé à Llanchu, mais s'est maintenue dans les communautés voisines qui possèdent encore des terres communales. Ce type de compétition agricole semble fort répandu dans les Andes. Attestée dans la région de Cusco, elle l'est également dans les Andes centrales du Pérou par exemple (Caroline Magny, communication personnelle). Les hommes et les femmes de la communauté commentent les aptitudes au travail de chacun à l'aune de ses capacités à sarcler les sillons. De façon littérale comme figurée, un homme doit «faire son sillon » (que. wachu) pour gagner le « respect» (esp. respecto) des autres.

D'un point de vue technique, la reconstitution de sillons permet d'irriguer la parcelle qui n'a bien souvent pas reçu une goutte d'eau durant le mois écoulé. Ces irrigations d'appoint sont très différentes de la première : moins d'eau est utilisée et, le flux d'eau étant souvent assez important, il faut savoir anticiper, réagir vite et estimer avec précision sa force. Si l'eau déborde d'un sillon, celui-ci ainsi que ceux situés en-dessous auront tendance à s'écrouler rapidement, des plants seront déracinés et l'irrigation du sillon sera plus difficile. L'eau doit être guidée méthodiquement de raie en raie. Les rigoles de la chakapa ou le killan (situé au centre) servent à diriger l'eau vers les sillons en contrebas. Avec cette technique, toute l'eau reste dans la parcelle car il n'y a pas de système de drainage. Quand elle arrive au bout du sillon, elle est redirigée vers un nouveau sillon encore sec. Lors de ces irrigations d'entretien, les raies (que. wachu), les bords (que. chakapa) et les saignées (que. killan) deviennent des aménagements indispensables.

À propos de ces irrigations complémentaires, les paysans n'hésitent pas à signaler des ressemblances entre irrigation et tissage. D'après Alberto Yupanqui (41 ans, évangélique, 2005), la chakapa est la matérialisation même du fait que la parcelle est comme un textile. Elle empêche l'eau de s'échapper de la parcelle comme la lisière empêche les fils de se défaire. Or les tissus andins sont systématiquement renforcés par un galon ou une broderie représentant souvent un motif aquatique. De leur côté, Arnold, Yapita et Apaza (1996, p. 378) ont également constaté l'importance accordée par les paysans aux bordures qui font, selon eux, de la parcelle un textile, mais plus encore lui donnent vie en devenant un corps, une personne (esp. fórmase gente; aym. jaqichana). Curieusement, l'agriculture dont nous parlent ces auteurs n'est pas irriguée et ne nécessite pas de chakapa. Cela n'empêche pas que la bordure y soit un élément primordial. D'autres éléments textiles, pour lesquels nous avions identifié des correspondances avec des aménagements agricoles, semblent liés à l'idée d'une circulation hydraulique. 
Nous avons vu le parallèle entre les bandes de couleurs pleines (esp. listas) et les raies dans lesquelles s'écoule l'eau durant les irrigations complémentaires à Llanchu. Les femmes de Tarabuco (Bolivie) procèdent à la même identification : elles appellent les bandes parallèles de leurs jupes llut'asqa (que.), terme qui signifie littéralement " celui qui ne laisse pas se déverser, est bouché, obstrué » (Cereceda et al. 1993, p. 24). En relevant les ressemblances que les paysans voient entre les techniques agricoles et le tissage, nous avons pu montrer l'importance du bord de la parcelle et celle, dans une moindre mesure, des sillons et de la rigole centrale. Puisque la parcelle retient l'eau qu'elle reçoit, il faut la considérer comme une unité hydraulique. Au-delà de l'analogie entre la parcelle et le textile, la première peut également être comparée à un être vivant, voire à un être humain.

L'analogie entre humain et végétal joue sur deux niveaux complémentaires, celui de la parcelle et celui de chaque plante. Au fur et à mesure des irrigations, les plantes croissent dans la parcelle, se nourrissant de la terre comme un embryon se nourrit du sang de sa mère ${ }^{26}$. Lestage $(1999$, p. 35$)$ montre que « la langue quechua fait correspondre les moments essentiels du cycle vital humain aux étapes de la croissance d'une plante : maturation du fruit (grossesse), fruit tendre (jeune enfant), fruit mûr (adulte), plante desséchée (maladie, puis mort) ». Étendant sa recherche dans le temps et dans l'espace et en s'intéressant aux différents dictionnaires quechua, elle note que, pour Cerrón Palomino, mallki signifie, dans la deuxième moitié $\mathrm{du} \mathrm{Xx}^{\mathrm{e}}$ siècle, à la fois « ancêtre momifié »et « pépinière, plante en pot, jeune plant ». Depuis le XVII ${ }^{\mathrm{e}}$ siècle jusqu'à aujourd'hui, un rapprochement est fait dans la langue quechua entre le « mûrissement » et la croissance des individus. Ainsi un enfant est-il « vert, tendre » (que. llullu), tandis qu'un homme d'expérience est « mûr » (que. poccosa runa) (ibid., citant González Holguín). À Llanchu, la notion de « mûrissement » est importante, à l'instar de celle de « floraison » (esp. florecimiento), ce qui rend l'analogie plus explicite. Lors de leur mariage, les jeunes femmes « fleurissent » (que. tikashanku) et Margarita (53 ans, évangélique, 2005) assure que « nous sommes comme les fleurs, on fleurit et puis après on se dessèche ». Cette notion de "floraison » a été soulignée par plusieurs auteurs travaillant dans différentes régions des Andes (Vokral 1991, p. 320 ; Arnold in Jiménez et Yapita 1996, p. 186) ; elle peut aussi être appliquée à d'autres domaines, par exemple les animaux, les commerces où on dit que les initiatives personnelles et collectives « fleurissent ». Ainsi, l'analogie entre reproduction végétale et reproduction humaine englobe les différentes étapes de croissance des végétaux et des êtres humains.

\section{Récolte et stockage}

Les deux dernières étapes de la récolte et du stockage fournissent, elles aussi, des éléments intéressants. Dans le cas du maïs, les pieds sont coupés à environ $20 \mathrm{~cm}$ du sol, les épis sont mis à sécher au soleil, tandis que les tiges sont données 
au bétail (fraîches ou séchées). Les pommes de terre sont déterrées à la houe. La récolte est ensuite placée sur des mantas (carrés de tissu ou de plastique) qui permettront son transport à dos d'homme. Au moment de la récolte, il est important de respecter certaines conventions. Dominga (61 ans, évangélique, 2004) raconte comment son père travaillait. Une fois la pomme de terre extraite, il prenait bien soin de frotter chaque tubercule avant de les empiler les uns sur les autres (l'apu de la communauté de Llanchu s'appelle justement phinaysuni, le grand amoncellement [de pommes de terres]). Puis il faisait brûler de la paille à proximité, de telle sorte que la fumée atteigne la récolte. Après avoir sorti de la réserve les tubercules de l'année précédente, il stockait les pommes de terre nouvelles avec cérémonie, pieds nus et il exécutait un rite qui avait pour but de faire en sorte que les pommes de terre soient bien conservées et qu'elles soient en quantité suffisante pour l'année à venir. La récolte était de nouveau enfumée, en brûlant cette fois de la graisse d'alpaga, dans l'espace de stockage. Il prenait soin de mettre un taqe, une pomme de terre à trois têtes, au-dessus du tas ; celui-ci devait « accrocher » l'animu et le transmettre à l'ensemble des tubercules pour qu'ils soient forts et se conservent bien (no se acaben). Rodrigo Hancco (41 ans, catholique, Accha Baja, 2005) explique que le taqe est une manifestation de l'animu de la plante et qu'il sert d'accroche. Les divinités masculines (apu) et féminine (Pachatierra) sont donc sollicitées pour protéger la récolte.

\section{À LA RECHERCHE D’UNE LOGIQUE ANALOGIQUE}

Pour essayer de dégager la logique qui sous-tend l'exposé ci-dessus, nous utiliserons le concept de "schème intégrateur de la pratique » que Bourdieu (1980) a exposé brièvement dans son ouvrage Le sens pratique. Pour cet auteur, ce concept « importe dans l'action », mais il "n'est ni un "plan" consciemment établi à l'avance qu'il suffirait d'exécuter [...], ni un "inconscient" qui orienterait mécaniquement la pratique » (ibid., p. 348). Il a pour grand avantage de prendre en compte la " pratique » dans laquelle nous incluons les techniques et les savoirs sur l'environnement, à l'instar de ce que proposent Lakoff et Jonhson (1985) ou Latour et Lemonnier (1994) ou Lemonnier (1994). Dans leur article conjoint, Latour et Lemonnier (1994, pp. 20-21) écrivent que « les mêmes mots qui sonnent comme des métaphores [...] passent en effet, par transfert, transport et traduction, du domaine de l'action sur la matière au domaine de l'action sur le corps social ou le corps propre [...] on obtient alors un langage commun, à double sens, où les propriétés acquises dans un domaine seraient réemployées dans l'autre ». Dans ce cadre, comme l'indique Descola (2005, p. 286), l'analogie « traite des similitudes non pas entre les choses elles-mêmes, mais entre les relations qu'elles entretiennent, dispositif souple et polyvalent de production de ressemblance ».

Les travaux de ces auteurs incitent à envisager les analogies comme générées par un schème, mental mais également incorporé dans la pratique. Largement 
implicite, le schème guiderait non seulement la perception que les hommes ont de leur environnement, mais aussi leurs actions. Dispositif souple, il permettrait de réagir dans une multitude de situations, notamment en cas de difficulté. Cependant, il ne fonctionnerait qu'imparfaitement. Il n'est pas nécessaire que les correspondances soient exactes jusque dans les moindres détails. Voyons, dans le cas qui nous intéresse, si nous pouvons dégager un schème de ce type. L'ethnographie qui précède a permis d'identifier des correspondances entre agriculture et physiologie humaine, entre agriculture et textile. Reprenons-les séparément.

\section{Physiologie humaine et physiologie végétale}

Il est clairement apparu que la reproduction humaine sert de modèle pour concevoir la reproduction végétale. Deux éléments principaux sont à retenir. D'une part, la reproduction végétale est sexuée, d'autre part, les plantes sont dotées d'une énergie de même nature que celle des êtres humains et des entités surnaturelles incarnées dans le paysage. Selon l'ordre chronologique imposé par le cycle agricole, un couple - la Pachamama et les apu - est marié le $1^{\text {er }}$ août, quand les paysans considèrent que la terre est ouverte et a faim. Puis, lors de la première irrigation, les substances des époux se rencontrent, la Pachamama est fécondée par un apu, comme c'est le cas pour une femme et un homme. Enfin, lors du semis, les graines viennent occuper la place des embryons dans la terre (Figure 8). Les rituels ne célébreraient donc pas trois mariages différents, mais scanderaient trois étapes del'union de deux partenaires.

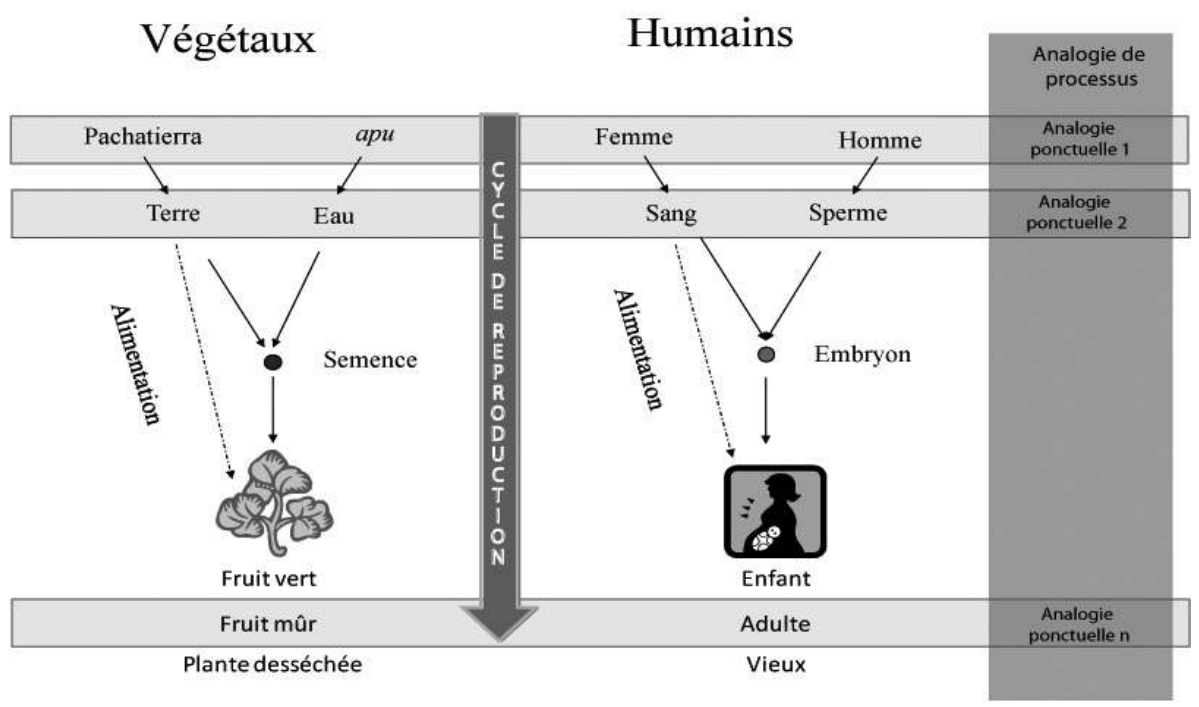

FIG. 8 - Analogie entre reproduction végétale et reproduction humaine (dessin Hall). 
L'eau s'avère être extrêmement importante. En effet, pour les paysans, la fertilité est liée à la présence d'eau. Celle-ci, retenue dans la terre cultivée, peut être assimilée au sang qui ne s'écoule plus du corps de la femme enceinte (La Riva 2000 , p. 175). Les paysans font un parallèle entre la reproduction humaine et la reproduction végétale : nous parlerons d'une analogie de processus.

\section{Techniques agricoles et tissage}

L'autre grand domaine mobilisé, par analogie, lors de la mise en culture d'une parcelle irriguée est celui du textile. Dans ce cas, deux processus, techniques cette fois, sont mis en relation. L'analogie fonctionne sur des niveaux complémentaires qu'il est nécessaire de distinguer.

Tout d'abord, les techniques agricoles sont associées au tissage par les paysans. La terre doit être travaillée et bien nettoyée, tout comme la laine doit être débarrassée des débris et des impuretés pour être démêlée, puis filée. À cette première phase de préparation du fil succède le tissage lui-même. Les deux derniers labours ( primera et segunda reja) sont mis en parallèle respectivement avec l'ourdissage, puis le tissage. Le travail du pourtour finit l'ouvrage en fermant la parcelle, comme le travail des bords pour un tissage permet de renforcer ses lisières. Comme pour l'analogie avec la physiologie humaine, il s'agit d'une analogie de processus (Figure 9). Ensuite, lors des irrigations d'appoint, les paysans considèrent que l'homme en guidant l'eau reproduit les gestes du tissage. On peut donc identifier une seconde analogie de processus entre irrigation et tissage, laquelle est périodiquement activée.

\begin{tabular}{|l|l|}
\hline $\begin{array}{l}\text { TECHNIQUES AGRICOLES } \\
\text { Labours et nettoyage de la parcelle }\end{array}$ & $\begin{array}{l}\text { TECHNIQUES TEXTILES } \\
\text { Nettoyage de la laine, démêlage des fibres } \\
\text { Filage de la laine }\end{array}$ \\
$\begin{array}{l}\text { Derniers labours } \\
\text { Primera reja }\end{array}$ & $\begin{array}{l}\text { Ourdissage du métier à tisser } \\
\text { Tissage }\end{array}$ \\
Semis & Reja reja \\
Travail des bords de la parcelle, chakapa & Renfoment des lisières du textile \\
\hline
\end{tabular}

FIG. 9 - Analogie de processus entre techniques agricoles et textiles.

Enfin, nous avons vu que les sillons renvoient aux bandes de motifs, les raies aux listas ou bandes de couleur sans motifs, la rigole centrale à la couture entre les deux laizes et la chakapa aux broderies ou galons qui viennent renforcer les bords du textile (Figure 10). L'analogie porte sur la structuration interne de la parcelle et du textile. Nous parlerons cette fois d'une analogie de structure. 


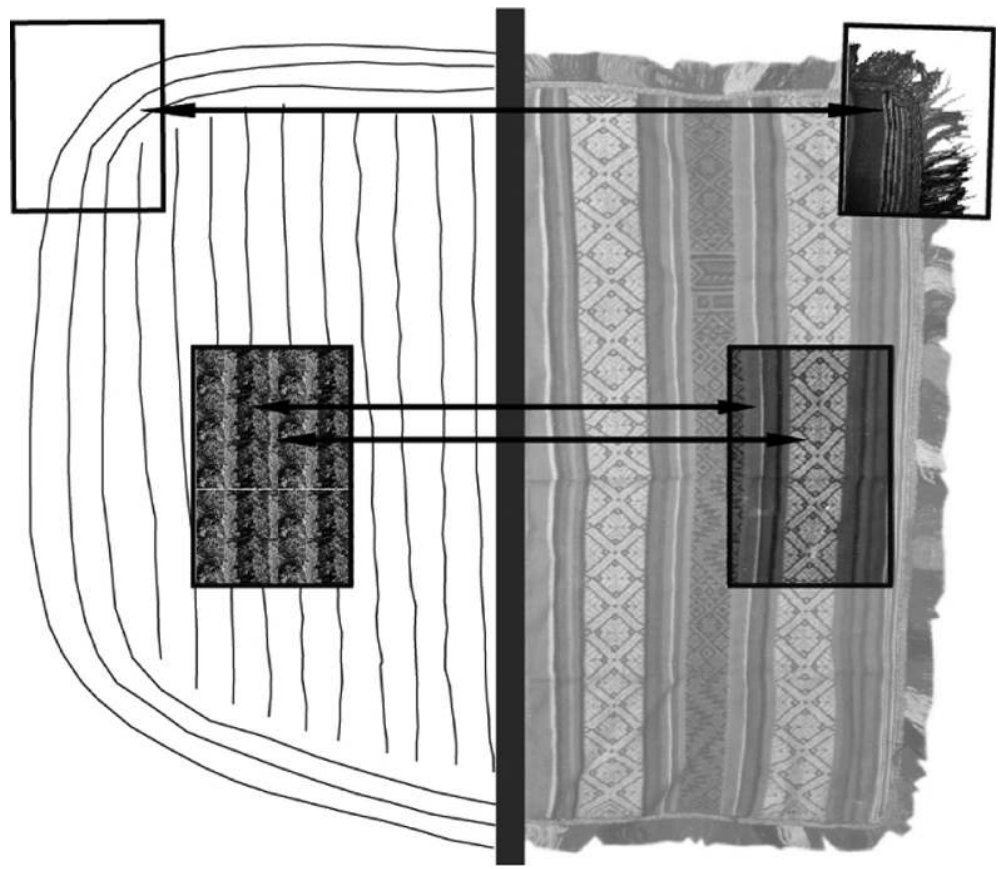

FIG. 10 - Analogie de structure entre une parcelle et une pièce textile (dessin Hall).

Le parallèle entre irrigation et textile peut être décomposé en trois analogies, une de structure et deux de processus. L'analogie de structure est maintenue tout au long du cycle agricole tandis que les analogies de processus se succèdent dans le temps.

\section{Le schème générateur de la pratique}

S'il a été nécessaire de distinguer ces analogies pour les besoins de l'analyse, à présent il faut les considérer ensemble car elles se renforcent mutuellement sur plusieurs points. Si chacune d'entre elles ne rend que partiellement compte du cycle agricole, ensemble elles permettent de mettre au jour une logique simple, un schème pour reprendre le vocabulaire de Bourdieu. Voyons sur quels points les analogies entre grands domaines sémantiques se recoupent. Tout d'abord, dans chaque domaine d'application, le moment du semis constitue un moment de transition important. Il marque le début du cycle végétatif des nouvelles plantes. Dans le champ de la physiologie humaine, il correspond au moment où les substances entrent en contact et vont former l'embryon. Du coup, une fois cette étape passée, il n'est plus question du couple formé par une entité féminine et une 
autre masculine, mais de la relation mère-enfant. Dans le textile, ce moment correspond au début du tissage. On peut en conclure qu'il existe une similitude dans la chronologie des actions dans les trois domaines analysés.

Ensuite, il est apparu que les limites sont particulièrement importantes : la chakapa retient les plantes et l'eau dans la parcelle, le tissage des quatre lisières renforce et protège l'intégrité de la pièce textile, le corps de la femme en période de gestation garde le sang menstruel. Cette insistance sur les limites permet de souligner en réalité la cohérence et l'indivision de l'unité. Nous avons vu que, par le biais de la physiologie humaine, il est possible d'expliciter le processus de reproduction végétale. Cette fois, par le biais du tissage, on relève l'importance de l'unité physique pour le tissu, pour la parcelle ou la plante, pour le corps humain enfin. Finalement il ressort que, pour les paysans, un être vivant est une unité physique qui contient de l'énergie vitale à l'instar d'une parcelle, d'une plante et d'une pièce textile ${ }^{27}$.

\section{Conclusion}

À partir de l'ethnographie présentée ici, nous avons constaté que les paysans andins établissent plusieurs analogies entre les plantes, les êtres humains et les pièces textiles. Ce premier constat ne rend pas compte de la complexité de la logique qui sous-tend ces correspondances. En effet, les analogies relevées - entre physiologie végétale et physiologie humaine, entre labour et tissage, entre irrigation et tissage, enfin entre parcelle et textile - permettent de mettre en évidence à la fois la conception anthropomorphe de la reproduction végétale et l'importance de « bien fermer » la parcelle à l'instar d'un textile andin. Ces analogies partielles doivent donc être considérées dans leur ensemble et non pas de façon isolée.

Par ailleurs, nous avons pu distinguer deux types d'analogies, de structure et de processus. Dans ce dernier cas, on a noté que, pour un processus qui évolue dans le temps, interpréter les correspondances terme à terme est contre-productif, voire source d'erreurs. En effet, à chaque étape du processus, les termes mis en correspondance évoluent : l'eau d'irrigation assimilée à du sperme, qui féconde la terre dans un premier temps, sera ensuite perçue comme du sang menstruel qui alimente les plantes. De masculine, l'eau devient féminine. Ainsi, les analogies doivent-elles être envisagées de façon dynamique et non statique. De plus, si on se place du point de vue des paysans, ces analogies prennent un sens pragmatique, elles permettent de résoudre et de comprendre toutes sortes d'événements. La circulation des fluides corporels sert de modèle interprétatif pour appréhender l'environnement et la fertilité végétale. Pour revenir sur l'idée du schème (conçu comme un plan d'action inconscient), celui-ci permet de donner du sens aux actes et de prévoir le type d'action à mener par une « généralisation pratique de tous les 
problèmes de même sorte » (Bourdieu 2000, p. 333). C'est ainsi que le schème est « générateur de la pratique » (Bourdieu 1980) et qu'il répond aux exigences de souplesse et d'adaptabilité mises en évidence par plusieurs auteurs (Lévi-Strauss 1962 ; Bourdieu 1980, 2000 ; Lakoff et Jonhson 1985 ; Bloch 1995 ; Descola 2005).

On peut aller plus loin et associer l'adjectif « intégrateur » à ce concept, ainsi que l'a fait Descola (2005, p. 162). Cela renvoie alors à «l'intégration de soimême par rapport à autrui » (ibid.). Autrement dit, ce schème permet à l'homme de se situer par rapport aux autres existants, avec lesquels il cohabite, comme les plantes, les animaux, les montagnes, la terre ou encore les objets (les pièces de textiles, par exemple). En parlant de l'agriculture en termes de reproduction sexuée conçue sur le modèle humain, l'homme reconnaît qu'il est porteur de la même énergie vitale que ces autres existants. L'homme ne se distingue pas fondamentalement de ces non-humains, il se considère, au contraire, comme faisant partie intégrante de la nature. D'après l'étude du cycle agricole, une hiérarchie entre ces existants est apparue. Les entités surnaturelles incarnées dans les montagnes et la terre ( $a p u$ et Pachatierra) octroient leur force vitale aux hommes pour qu'ils obtiennent de bonnes récoltes qu'ils conserveront longtemps. Entre ces partenaires doit être instaurée une relation de réciprocité, l'homme doit honorer les divinités pour qu'elles insufflent leur énergie aux plantes. Si un homme veut une bonne récolte, il doit non seulement établir une relation de réciprocité avec les entités surnaturelles, mais également savoir bien diriger cette énergie vers sa parcelle et ses plantes. L'homme se trouve en position de médiateur et les actions techniques sont empreintes d'un sens moral et symbolique.

Finalement, ces analogies ne peuvent pas être considérées seulement de façon explicite, elles contiennent une part implicite qui se manifeste dans la pratique rituelle et/ou technique. Dans ce cas, la pratique devient porteuse de sens. Celui-ci n'est exprimé qu'imparfaitement et indirectement par les mots et les idées. C'est ce que nous avons vu quand nous avons abordé l'homologie classificatoire de la terre et du sang. Ce «sens pratique » permet d'inscrire le symbolique dans le banal, le quotidien, le pragmatique. *

* Manuscrit reçu en décembre 2009, accepté pour publication en juin 2011.

\section{Notes}

Je tiens à remercier pour leur aide François Sigaut, Sophie Desrosiers, Antoinette Molinié, Jacques Galinier ainsi que Caroline Magny et Federica Tamarozzi. Leurs conseils m'ont aidée à élaborer le présent travail.

1. Ce travail de terrain a donné lieu à la rédaction d'une thèse (Hall 2009).

2. Dans le monde andin, Cereceda (1978) a été la première à analyser ces analogies de façon 
systématique. En proposant une lecture structurale des pièces textiles, elle a influencé les travaux ultérieurs sur les structures sociales et/ou spatiales des communautés andines (Molinié 1996), sur la musique (Martínez 1990)... Cereceda (1987) s'est également intéressée à ces analogies à travers le champ de l'esthétique. Les références au textile, si fréquentes, laissent même supposer à quelques auteurs que le tissage constitue une sorte de « matrice » fondamentale de la culture andine (Arnold 1996, p. 22). L'agriculture est une autre grande activité qui a été explorée dans ce sens, elle a souvent été mise en parallèle non seulement avec le textile (Silverman 1994; Seibold 1992), mais aussi avec la physiologie humaine (Bastien 1985). Sur ce sujet, les travaux d'Arnold (2000) en Bolivie, ainsi que l'ouvrage collectif plus ancien qu'elle dirigea avec Yapita (1996), sont sans aucun doute les plus complets d'un point de vue ethnographique. Ils tendent à montrer la densité des analogies, leur caractère à la fois redondant et polymorphe.

3. Si un tiers seulement des terres agricoles est irrigué, elles produisent l'essentiel des denrées commercialisées. La faible surface de terres irriguées est liée à des contraintes agronomiques, l'irrigation n'étant intéressante qu'au-dessous de $3800 \mathrm{~m}$ d'altitude, tandis qu'au-dessus, les gels trop fréquents sont néfastes pour la survie des plantes. Les deux cultures pratiquées sous irrigation sont principalement le maïs (blanco ou amarillo) et les pommes de terre (maway, blanca surtout).

4. À Llanchu, les « évangéliques » sont des personnes converties à une église dite " protestante », l'église évangélique péruvienne.

5. Notons que, dans les communautés voisines de Llanchu, l'influence des églises protestantes n'est pas aussi forte et ces rituels sont encore pratiqués.

6. Les membres de la communauté de Llanchu sont de langue maternelle quechua, mais parlent également l'espagnol. Les entretiens ont majoritairement été menés en espagnol, mais le quechua était toujours présent, aussi les termes indiqués dans le texte seront-ils tantôt en espagnol, tantôt en quechua. Pour le lecteur, la langue sera spécifiée par les abréviations respectives « esp. » et « que. ». Pour les termes qui sont un mélange espagnol et quechua, nous utiliserons «esp./que. ». Enfin, pour l'aymara, on mettra « aym. ».

7. Le nom, l'âge et la confession religieuse de chacun de mes interlocuteurs seront systématiquement précisés, les deux dernières précisions étant importantes pour analyser précisément leurs propos.

8. Étant donnée la façon labile d'indiquer le pluriel en quechua, j'ai fait le choix de ne pas marquer le pluriel sur le terme $a p u$.

9. L'utilisation du terme " divinité » pourrait être discutée, mais ce n'est pas ici le lieu d'un tel débat. Pour une discussion sur la nature des «divinités » andines et sur leur genre, voir notamment Allen (2002, chap. I).

10. Valderrama et Escalante (1988, pp. 82-83) retrouvent cette même classification des terres dans la vallée du Colca. Un mythe relevé par Cáceres (1986, p. 111) à Ccorca (Anta, Cusco) fait également apparaître ces trois types de sol, et seulement ceux-là. Dans le récit que cet auteur rapporte, le couple protagoniste s'alimente de ces trois types de terre. Cette classification semble donc non seulement dépasser les frontières de Llanchu, mais également avoir une portée mythologique.

11. Cette classification a également été signalée par La Riva (2005, p. 75).

12. L'accent quechua en espagnol rapproche le terme anemia de celui d'animia, mais il semble qu'il ne s'agisse pas seulement d'une déformation, le sens même du mot est altéré.

13. Il n'y a pas d'association explicite et directe entre ces deux substances, personne ne dira en effet que le sang et la terre sont équivalents. En revanche, l'homologie classificatoire permet de mettre en évidence l'existence d'un lien fort entre les deux.

14. De nombreux auteurs travaillant sur des rituels d'irrigation très élaborés réalisés dans les Andes centrales, par exemple Gelles (2000, p. 78) ou Valderrama et Escalante (1988), expliquent que l'eau est assimilée à du sperme, du sang ou, même, de l'urine ou du lait (ibid., p. 101).

15. Comme l'indique ce nom espagnol et nationaliste, cette appréciation des pommes de terre farineuses semble généralisée au Pérou et, plus largement, dans les Andes, d'après nos informations.

16. Notons qu'un élément résiste à l'interprétation : une fois cuits, ces embryons ne peuvent pas se développer. 
17. Plusieurs données boliviennes montrent l'importance de la « bonne torsion » pour une série de processus relevant de domaines différents, allant du textile (Cereceda 1987) à la musique (Martínez communication personnelle, 2007) en passant par la conception de l'être humain (Platt 2002, p. 10) ou, encore, la métallurgie (Absi 2003, p. 101). Cela donne à penser qu'il y a, peut-être, une différence culturelle entre la Bolivie et le Pérou.

18. À Qaqachaka en Bolivie, la terre mère est appelée « Mère Trame » (Mama Trama) lors de certains rites (Jiménez et Yapita 1996, pp. 142-147). Pour un des habitants, « la Mama Trama c'est la terre elle-même » (Arnold, Yapita et Apaza 1996, p. 375).

19. En Bolivie également, Arnold, Yapita et Apaza (1996, pp. 378-380) constatent que les bordures des pièces de textile sont comparées à celles de la parcelle.

20. Ce sac appartient à la collection personnelle de Sophie Desrosiers qui l'a acheté à La Paz dans les années 1980. Je la remercie de me l'avoir montré et de m'avoir fourni cette photographie.

21. Pièce textile carrée que les femmes portent sur leurs épaules.

22. Pièces carrées utilisées pour porter sur le dos les enfants, de l'herbe coupée... Auparavant tissées par les femmes, celles-ci sont dorénavant produites industriellement et vendues sur les marchés.

23. Notons que la partition de la parcelle et de la pièce textile ne se fait pas dans la même direction : tandis que les sillons sont perpendiculaires à la rigole centrale, les bandes de motifs sont parallèles à l'axe central.

24. Sur d'autres terrains, l'analogie entre espace agricole et pièce textile est envisagée à l'échelle du territoire communal (Jean Bourliaud, communication personnelle 2006 ; Silverman 1994, pp. 56-60 ; Molinié 1996, p. 221).

25. Notons que les entités supérieures féminines seraient multiples, comme les entités masculines.

26. Cette représentation de la grossesse vaut aussi bien à Llanchu qu'à Surimama (La Riva 2000, p. 171) ou chez les Macha de Bolivie (Platt 2002).

27. Voir l'article de Desrosiers (2000) dont le titre, en particulier, est tout à fait explicite.

\section{RÉFÉRENCES CITÉES}

Absi Pascale

2003 Les ministres du diable. Le travail et ses représentations dans les mines de Potosí, Bolivie, L'Harmattan, Paris.

Allen Catherine J.

2002 The hold life has. Coca and cultural identity in an Andean community, Smithsonian Institution Press, Washington/New York [1988].

ARNOLD Denise

1996 «Introducción », in Denise Arnold et Juan de Dios Yapita (éd.), Madre Melliza y sus crías = Ispall mama wawampi : antología de la papa, Hisbol/Ediciones ILCA, La Paz, pp. 1-28.

2000 " "Convertirse en persona" el tejido : la terminología aymara de un cuerpo textil », in Victòria Solanilla Demestre (éd.), Actas de la I Jornada internacional sobre textiles precolombinos, Universitat autònoma de Barcelona, Barcelone, pp. 9-28.

Arnold Denise et Juan de Dios Yapita (éd.)

1996 Madre Melliza y sus crías = Ispall mama wawampi : antología de la papa, Hisbol/Ediciones ILCA, La Paz. 
Arnold Denise, Juan de Dios Yapita et Cipriana Apaza

1996 «Mama Trama y sus crías : analogías de la producción de la papa en los textiles de Chuquiñapi, Bolivia », in Denise Arnold et Juan de Dios Yapita (éd.), Madre Melliza y sus crías = Ispall mama wawampi : antología de la papa, Hisbol/Ediciones ILCA, La Paz, pp. 373-411.

BASTIEN Joseph

1985 «Qollahuaya-Andean body concepts : a topographical-hydraulic model of physiology », American Anthropologist, 87 (3), pp. 595-611.

BLOCH Maurice

1995 «Le cognitif et l'ethnographique », Gradhiva, 17, pp. 44-61.

BOURDIEU Pierre

1980 Le sens pratique, Éditions de minuit, Paris.

2000 Esquisse d'une théorie de la pratique, Seuil, coll. « Point», Paris [1972].

BOYer Pascal

1993 "Cognitive aspects of religious symbolism», in Pascal Boyer (éd.), Cognitive aspects of religious symbolism, Cambridge University Press, Cambridge, pp. 4-47.

\section{CÁCERES Efraín}

1986 «El agua como fuente de vida. Tradición y escape en los mitos andinos », Allpanchis, 28, pp. 99-122.

Cereceda Verónica

1978 «Sémiologie des tissus andins : les talegas d'Isluga », Annales ESC, 33 (56), pp. 1017-1035.

1987 " Aproximaciones a una estética andina », in Thérèse Bouysse-Cassagne, Olivia Harris, Tristan Platt et Verónica Cereceda, Tres reflexiones sobre el pensamiento andino, Hisbol, La Paz, pp. 133-231.

Cereceda Verónica, Johnny Dávalos et Jaime Mejía

1993 Una diferencia, un sentido : los diseños de los textiles Tarabucos y Jalq'a, ASUR, Sucre.

Classen Constance

1993 Inca cosmology and the human body, University of Utah Press, Utah.

Descola Philippe

2005 Par delà nature et culture, Gallimard, coll. «Bibliothèque des Sciences Humaines », Paris.

DesRosiers Sophie

2000 «Le tissu comme un être vivant ? À propos du tissage à quatre lisières dans les Andes ", in Françoise Cousin, Sophie Desrosiers, Danielle Geirnaert et Nicole Pellegrin, Lisières et bordures. Actes des premières journées d'étude de l'association française pour l'étude du textile (Paris, 13-14 juin 1996), Les Gorgones, Bonnes, pp. 117-125.

GeÉLles Paul H.

2000 Water and power in Highland Peru. The cultural politics of irrigation and development, Rutgers University Press, New Brunswick/Londres. 
Griaule Marcel

1975 Dieu d'eau, Fayard, Paris [1948].

HALL Ingrid

2009 De la loi à l'esprit. Ethnogenèse récente d'une communauté paysanne des Andes sud péruviennes, thèse de doctorat, Université Paris Ouest Nanterre La Défense, Nanterre.

HAUdricourt André-Georges

1962 « Domestication des animaux, culture des plantes et traitement d'autrui », L'Homme, 2 (1), pp. 40-50.

JiMÉNEZ Domingo et Juan de Dios YAPITA, avec commentaires de Denise ArNold

1996 "La metafísica de la papa », in Denise Arnold et Juan de Dios Yapita (éd.), Madre Melliza y sus crías = Ispall mama wawampi : antología de la papa, Hisbol/Ediciones ILCA, La Paz, pp. 139-193.

La Riva GonzÁLEz Palmira

$2000 \quad$ "Le walthana hampi ou la reconstruction du corps. Conception de la grossesse dans les Andes du sud du Pérou », Journal de la Société des Américanistes, 86, pp. 169-184.

2005 «Las representaciones del animu en los Andes del Sur peruano », Revista andina, 41, pp. 63-88.

LAKOFF Georges et Mark JoHnson

1985 Les métaphores dans la vie quotidienne, Éditions de minuit, Paris.

LAtour Bruno et Pierre Lemonnier (éd.)

1994 De la préhistoire aux missiles balistiques: l'intelligence sociale des techniques, La Découverte, Paris.

LEMONNIER Pierre

1994 "Choix technique et représentations de l'enfermement chez les Anga (Papouasie Nouvelle-Guinée). Ethnologie et technologie », in Bruno Latour et Pierre Lemonnier, De la préhistoire aux missiles balistiques, La Découverte, Paris, pp. 253-272.

LeSTAGe Françoise

1999 Naissance et petite enfance dans les Andes péruviennes, L'Harmattan, Paris.

LÉVI-STRAuss Claude

1962 La pensée sauvage, Plon, Paris.

1991 La potière jalouse, Presses Pocket, Paris [1985].

Martínez Rosalía

1990 «Musique et démons : carnaval chez les Tarabuco (Bolivie) », Journal de la Société des Américanistes, 76, pp. 155-176.

Marzal Manuel

1971 « ¿ Puede un campesino cristiano ofrecer un "pago a la tierra"?», Allpanchis, 3, pp. 116-129. 
MoliniÉ-Fioravanti Antoinette

1996 "The spell of Yucay : a symbolic structure in Incaic terraces », Journal of the Steward anthropological society, 24 (1-2), pp. 203-230.

PÉREZ GaLÁn Beatriz

2004 Somos como incas. Autoridades tradicionales en los Andes peruanos, Iberoamericana, Cuzco/Madrid, Vervuert, Francfort-sur-le-Main.

Platt Tristan

2002 «El feto agresivo. Parto, formación de la persona y mito-historia en los Andes », Estudios Atacameños, 22, pp. 127-155.

SEIBOLD Katharine E.

1992 «Textiles and cosmology in Choquecancha, Cuzco, Peru », in Robert E. Dover, Katharine E. Seibold et John Holmes McDowell (éd.), Andean cosmologies through time : persistence and emergence, Indiana University Press, Bloomington, pp. 167-201.

SiLVERMAN Gail

1994 El tejido andino : livro de la sabiduría, Fondo editorial central de reserva del Perú, Lima.

VALDERrama Ricardo et Carmen Escalante

1988 Del Tata Mallku a la Pacha Mama. Riego, sociedad y ritos en los Andes peruanos, Desco, Lima.

VOKRAL Edite V.

1991 Qoñi-Chiri. La organización de la cocina y estructuras simbólicas en el Altiplano del Perú, Ed. Cotesu/Abya-Yala, Quito. 